\title{
Bertrand Auerbach (1856-1942), Éclaireur et « sans grade » de l'École française de géographie
}

Bertrand Auerbach (1856-1942), explorer and "lower-grade figure " of the French School of Geography

Bertrand Auerbach (1856-1942), Erkunder und « Randfigur » der französichen Schule der Geographie

\section{Marie-Claire Robic}

\section{OpenEdition}

\section{Journals}

Édition électronique

URL : http://journals.openedition.org/rge/4538

DOI : $10.4000 /$ rge.4538

ISSN : 2108-6478

\section{Éditeur}

Association des géographes de l'Est

Édition imprimée

Date de publication : 1 janvier 1999

ISSN : 0035-3213

\section{Référence électronique}

Marie-Claire Robic, «Bertrand Auerbach (1856-1942), Éclaireur et « sans grade » de l'École française de géographie », Revue Géographique de l'Est [En ligne], vol. 39 / 1 | 1999, mis en ligne le 08 septembre 2013, consulté le 08 septembre 2020. URL : http://journals.openedition.org/rge/4538 ; DOI : https:// doi.org/10.4000/rge.4538

Ce document a été généré automatiquement le 8 septembre 2020

Tous droits réservés 


\title{
Bertrand Auerbach (1856-1942), Éclaireur et « sans grade » de l'École française de géographie
}

\author{
Bertrand Auerbach (1856-1942), explorer and «lower-grade figure » of the \\ French School of Geography \\ Bertrand Auerbach (1856-1942), Erkunder und « Randfigur » der französichen \\ Schule der Geographie
}

Marie-Claire Robic

Bertrand Auerbach n'a pas marqué la mémoire disciplinaire, celle-ci ayant retenu, classiquement, l'œuvre d'un "fondateur» et de ses plus brillants «élèves" ou successeurs. Il a pourtant accompagné l'émergence de l'«École française» de géographie, une appellation qu'il utilisa dès $1908^{1}$. Enseignant à la faculté des lettres de Nancy durant quarante ans (1885-1926), titulaire à partir de janvier 1893 de l'une des premières chaires de géographie de l'Université française, il ne fut ni un éveilleur de vocations, ni le dénicheur et meneur d'hommes que l'un de ses cadets à l'École normale, Raoul Blanchard, incarna à Grenoble - foyer d'où essaimèrent nombre de géographes, tel Jules Blache, le successeur d'Auerbach à la chaire de Nancy après Robert Capot-Rey. Question de talent, mais aussi de personnalité: trop d'ironie et d'irrespect pour l'autorité, moindre ambition? Question de générations aussi. Entre Paul Vidal de la Blache, pionnier et en somme autodidacte en géographie, et la génération éduquée directement à la géographie moderne, la génération de B. Auerbach (1856-1942) est intermédiaire. Nous rappellerons, avec ses découvreurs successifs, les traits de l'un « des petits, des obscurs, des sans grade sans lesquels rien n'aurait été possible » dont André Meynier (1969) a déploré l'oubli. Nous tâcherons de comprendre à travers l'itinéraire d'Auerbach comment, alors, on se convertit de l'histoire à la géographie... Nous nous attacherons aussi à montrer en quoi Auerbach a accompagné l'affirmation de la géographie française dans deux de ses voies caractéristiques: l'insertion universitaire et le paradigme régional. Nous examinerons enfin en quoi sa personnalité, 
ses investissements propres, les liens entre ses intérêts de géographe et le milieu nancéien où il s'est implanté durablement le distinguent de ses codisciples.

\section{Sortir de l'obscurité}

2 La figure d'Auerbach est sortie de l'ombre depuis une vingtaine d'années. Il revient à Numa Broc (1974a) d'avoir exhumé ce "pionnier de la géographie en Lorraine ». Il retraçait pour la première fois sa vie et sa carrière ${ }^{2}$, soulignait ses thèmes de prédilection: étude des fleuves, géographie de la population, et surtout géographie régionale, Auerbach ayant publié, avec son livre sur Le Plateau lorrain, « la première monographie régionale de l'École française de géographie et [étant] sans doute le premier à avoir utilisé l'expression "géographie régionale"». N. Broc a souligné aussi sa parfaite connaissance de la science d'Outre-Rhin: Auerbach commentait les débats entre géographes allemands dans son cours inaugural en 1887 et, vingt ans après, dans une synthèse portant sur « les conceptions et les méthodes de la géographie »; brillant germanophone, il fut ainsi l'un des "grands intermédiaires » entre la France et la production géographique allemande (Broc, 1977).

De son côté, J.-C. Bonnefont a retracé le rôle joué par Auerbach dans la découverte géographique de la Lorraine et dans l'implantation de la discipline à la faculté des lettres de Nancy. On sait que Vidal de la Blache, tout jeune docteur, a occupé dès 1872 une nouvelle chaire "d'histoire et de géographie». Issue de la transformation de la chaire d'histoire ancienne transférée depuis Strasbourg, elle bénéficiait à la fois de la faveur des autorités, soucieuses de développer l'enseignement de la géographie, et de l'intérêt que les Nancéiens portaient à la géographie moderne, comme en témoigne le succès du cours assuré à la faculté des lettres, dès 1869 , par Léonce Pingaud ${ }^{3}$. Mais après le départ de Vidal pour l'École normale, début 1877, cette chaire mixte ne fut occupée durant une petite décennie que par des historiens qui comptaient bien le demeurer, et qui tous firent honneur à Cliot. Une « difficile succession » donc, jusqu'au bref passage de Marcel Dubois (1884-1885), happé par un poste de géographie coloniale à la Sorbonne, et jusqu'à son remplacement par B. Auerbach, qui y inscrivit toute sa carrière de géographe... J.-C. Bonnefont a relevé aussi deux aspects de la personnalité d'Auerbach que de trop rares documents permettent d'apprécier: un géographe " subtil et mal aimé » (Bonnefont, 1993a). Friand de la métaphore filée et de l'anecdote légère, Auerbach fut parfois mal apprécié de collègues et de supérieurs hiérarchiques qui lui reprochaient son manque de distinction ${ }^{5}$. Plus grave serait la condamnation proférée à son encontre par Vidal de la Blache à la fin de la guerre : mais, comme nous le verrons ci-dessous, nous pensons qu'il y a là un malentendu, Auerbach étant toutefois, probablement, en délicatesse avec l'expert-géographe que E. de Martonne a incarné durant la guerre.

4 La multiplication de travaux spécialisés permet de reconstruire finement l'état des lieux de la géographie à la fin du XIXe siècle. Aussi retrouve-t-on référence à Auerbach dans plusieurs ouvrages récents, dont l'anthologie des géographes français (Pinchemel et al., 1984). Gilles Palsky (1996) souligne combien il s'est préoccupé des problèmes méthodologiques posés par la représentation cartographique des densités. Olivier Soubeyran (1997) signale que, dans le conflit qui a opposé Lucien Gallois et Marcel Dubois, cofondateur des Annales de géographie avec Vidal de la Blache en 1891, Auerbach se situe du côté de Gallois: il est comme lui partisan d'une science pure, 
autoréférentielle, dont le modèle est la géographie des petits " pays ", alors que Dubois prône une discipline ouverte sur l'action et qu'il la met en œuvre dans la géographie coloniale. Marie-Vic Ozouf-Marignier et Marie-Claire Robic (1998) montrent que Auerbach, lecteur particulièrement perspicace du Tableau de la géographie de la France, s'est fait à la fois l'exégète de la géographie humaine émergente et l'interprète de la vertu décentralisatrice du livre de Vidal...

\section{Un historien normalien dans la promotion de la géographie à l'université}

5 Les décennies 1860-1890 furent une époque d'engouement pour la géographie propice à l'éveil de vocations ou plutôt de conversions à la géographie (Capel, 1982). Pour la France, on sait le lien qui a uni la promotion de la géographie à l'Université à sa diffusion dans les programmes scolaires, dans l'atmosphère de la Revanche. Loin de concerner la seule géographie, cette promotion participait de la politique scientifique volontariste par laquelle la République a affirmé sa propre conception positiviste de la formation du citoyen. Déjà présente ici et là à l'Université dans la fonction mineure de "géographie historique ", la géographie s'est autonomisée à partir de cette présence initiale, en s'émancipant de la tutelle historienne par un jeu subtil d'allégeance et de distanciation. Dans ce jeu, l'appui sur les sciences naturelles fut de grande utilité. C'était en même temps se détacher de la fonction utilitaire qu'assignaient à la géographie les lobbies les plus puissants qui en avaient fait la propagande. Parmi les promoteurs de cette voie universitaire, plusieurs générations de Normaliens furent au premier rang (Robic, 1993). Auerbach fait partie de ces générations normaliennes qui ont le plus bénéficié des réformes universitaires des débuts de la troisième République (Karady, 1973, p. 463 sq).

6 Entré à l'École normale en 1876, B. Auerbach appartient à une petit groupe situé entre la poignée des pionniers qui se sont engagés très tôt dans la promotion de la géographie et la cohorte de ceux qui soutiennent directement des thèses de géographie, illustrant d'emblée par leurs recherches une discipline moderne ${ }^{6}$. Or 1876-1877, c'est le moment où les Républicains accèdent au pouvoir. C'est l'époque même où la mutation de l'Université se produit, se transformant en un lieu de recherche et de formation de vrais étudiants (les bourses de licence et d'agrégation sont créées, de même que la maitrise de conférences). Pour une discipline comme la géographie s'ouvrent des espoirs de développement multiples, à la mesure de l'effervescence tout à la fois nationaliste, colonialiste et républicaine qui agite l'opinion. Aussi durant les décennies 1870-1890 il a fallu "s'improviser géographe», selon l'expression de Marcel Dubois (1888) et comme le faisait remarquer le jeune historien Debidour, candidat à la succession de Vidal en 1877: «Vidal-Lablache ne l'était pas non plus [géographe], quand on le prit à Angers, où il professait comme moi, en 1872, pour l'envoyer à Nancy $»^{7}$.

Or à la charnière soixante-dix-quatre-vingt, quelques élèves historiens, "futurs géographes » aux parcours contrastés se croisent à la rue d'Ulm : B. Auerbach a pour camarades de promotion M. Dubois (1856-1916) et Paul Dupuy (1854-1948). Ils n'y côtoient pas Lucien Gallois (1857-1941), intégré seulement en 1881, mais qui appartient à la même génération naturelle. Tous y rencontrent $\mathrm{p}$. Vidal de la Blache, qui prépare les élèves de troisième année à l'agrégation d'histoire et de géographie ${ }^{8}$. 
8 Licencié ès lettres en 1878, sorti en 1879 sans l'agrégation mais agrégé d'histoire et de géographie l'année suivante, Auerbach s'est orienté vers l'histoire dès la première année d'École ${ }^{9}$. Il paraît être un élève très apprécié pour ses qualités d'érudition et pour son don des langues (excellent germaniste, bon en anglais, très apprécié en grec, il sait aussi l'hébreu). L'enseignement de la géographie ne portait, avec Ernest Desjardins, que sur la géographie historique et, comme ses camarades, Auerbach ne semble pas avoir suivi l'enseignement de Vidal de la Blache avant la troisième année.

9 La vocation de géographe d'Auerbach ne paraît pas s'affirmer dans ses prestations de professeur de lycée (il est successivement affecté à Bourges, Laval, Belfort, Toulouse puis Lyon), ni dans ses premières années en université ${ }^{10}$. Il s'oriente vite vers un doctorat : il sollicite dès 1881 une bourse de voyage et demande en 1882 à être nommé dans une ville de faculté pour pouvoir entreprendre une thèse : "Privé de ressources bibliographiques et scientifiques, je n'ai pu que traduire le volume de l'Histoire grecque de Curtius (...) » déplore-t-il dans ses vœux. Nommé maître de conférences d'histoire ancienne à Caen en 1883, il obtient en 1885 une mission en Allemagne, où il consulte les archives de Dresde. Hormis des débuts délicats à Laval, ses supérieurs l'apprécient. Atout précieux, le tout-puissant L. Liard, vice-recteur de l'Académie de Paris et longtemps directeur de l'enseignement supérieur avait très bien noté le débutant du lycée de Bourges : « Intelligent, appliqué, l'autorité fait encore défaut » (1880).

Les choses ne changent qu'à partir de sa nomination à Nancy, où il accède en 1885 à la maîtrise de conférences d'histoire et de géographie. Les références à la géographie apparaissent alors dans son dossier régulièrement et se renforcent vite. Dès ses premières années le doyen et l'inspecteur Zeller commentent favorablement son activité : «Le séjour qu'il a fait en Allemagne l'a fortifié sans lui nuire. Il y est devenu érudit sans cesser d'avoir les qualités françaises» (1885-1986); «M. Auerbach est surtout un géographe instruit qui a de la méthode et beaucoup de lectures » (il a fait notamment un cours sur les Indiens d'Amérique du Nord). Son enseignement porte sur la géographie contemporaine (France, Europe centrale, États-Unis, région lorraine en 1890 et $1891 \ldots$..), sur des questions de géographie générale, dont l'ethnographie, sans oublier les auteurs au programme de l'agrégation : il aborde ainsi Strabon, Hérodote et Aristote ${ }^{11}$.

11 Dès 1891 le doyen Émile Krantz estime advenue une transformation : «À réussi à Nancy dans l'enseignement de la géographie, auquel il s'est adonné définitivement et où il serait juste de lui faire un avenir.» Trois ans plus tard, alors que la chaire a été spécialisée en géographie, il avise ainsi le recteur: "Monsieur Auerbach, qui était historien, est en train de devenir géographe. Il est juste de lui faire crédit de quelques années pour juger de sa compétence acquise.» Le dernier article d'histoire signé par Auerbach, avant longtemps, parait en 1889.

12 La fonction aurait-elle créé l'organe ? Ce n'est pas si simple car, à la différence des nombreux successeurs de Vidal à Nancy qui ont occupé ce poste comme une simple opportunité de carrière, Dubois et Auerbach semblent donc se réorienter en 1884-1885 vers la géographie. Par les thèses qu'il soutient en janvier 1888, Auerbach reste historien, "à cheval » toutefois sur les deux disciplines puisque sa thèse principale concerne l'histoire diplomatique tandis que la thèse latine porte sur la géographie de Strabon: il se conforme ici aux seuls genres reçus en Sorbonne, la géographie historique ou l'histoire de la géographie. La soutenance, difficile ${ }^{12}$, pourrait l'avoir contraint à la géographie, en une vocation forcée qui lui serait dictée par un jury fort 
critique sur ses performances d'historien. J.-C. Bonnefont rejette cette hypothèse à juste titre au vu du réel intérêt que Auerbach porte à la géographie moderne, comme l'atteste le succès de ses conférences publiques à l'Université et auprès de la Société de géographie de Nancy.

Mais sa compétence est à forger... Arguant de son impréparation autant que de la difficulté du sujet, Auerbach attend deux ans avant de présenter un cours inaugural de géographie à la rentrée 1887. Il y expose sa position, ancrée dans une analyse de l'histoire des sciences et dans un bilan des controverses qui agitent les géographes allemands. Autres manifestations de conversion, en 1888 il publie un «Buffon géographe » et il enchaîne dans la Revue de géographie une série d'articles sur la région lorraine.

14 En tout ceci, Auerbach se montre très sensible à la situation faite à la géographie en France et aux opportunités qu'elle ouvre. Il se révèle proche du groupe des Annales de géographie qui se constitue à partir du début des années 1890. Un événement s'est produit entre la nomination d'Auerbach sur un poste mixte fondé plus de vingt ans avant et le tout début des années quatre-vingt-dix: le ministère de l'Instruction publique a effectivement orienté le devenir de la géographie en l'inscrivant dans les facultés des lettres, avec éventuellement l'appoint de chaires de géographie physique sises en facultés des sciences, récusant en 1885 les tenants d'une École spéciale de géographie (Broc, 1974b).

C'est précisément cette solution universitaire que défend Auerbach lors de son cours inaugural en critiquant ouvertement les partisans de la solution opposée et en ridiculisant les prétentions des « dévots de la géographie ». De même, en écho (le cours de Auerbach est cité), M. Dubois (1888) avoue s'être rallié à la solution universitaire et au maintien de l'alliance histoire-géographie prônée par E. Lavisse, après avoir défendu la création d'une école spéciale. Tous deux fustigent les prétentions de néophytes imprudents et prônent la "patience» et "l'esprit scientifique» (Auerbach) ou le « tact » et la « discipline scientifique » (Dubois). L'Université leur paraît la plus propre à affronter leur programme rationaliste, pour des raisons intellectuelles et des raisons d'économie, puisque, rétorque Auerbach aux partisans d'une création ad hoc, «toutes les sciences auxiliaires y sont cultivées, depuis la géologie jusqu'à l'économie politique » $(1888$, p. 64). Et de rappeler le concours de son collègue Bleicher à une sorte d'» embryon de séminaire géographique » (id., p. 65).

Les choix d'Auerbach se conforment donc à la solution prônée par le Ministère en 1885 sous la pression de l'establishment universitaire parisien, et aux options qu'un petit groupe de normaliens très combatifs, regroupés autour de Vidal de la Blache, a réussi à faire admettre au Congrès international de géographie de Paris en $1889^{13}$, en se proposant d'établir autour de chaires de géographe implantées en facultés des lettres les «rapports organiques" avec les "sciences qui peuvent lui servir d'auxiliaires » (Robic, 1993).

\section{L'autonomisation du géographe moderne}

17 L'affirmation de cette voie universitaire va de pair avec l'autonomisation de la géographie par rapport à l'histoire. Effectivement, la spécialisation d'Auerbach et l'intérêt qu'il porte à la géographie moderne s'accusent, au détriment de ce qu'en attendraient ses collègues historiens. Ainsi dans sa notice individuelle de 1894, il est 
fait état de critiques relatives à sa conception de la géographie comme à sa désinvolture à l'égard des exercices canoniques de l'agrégation : «M. Auerbach est plutôt géographe qu'historien; on lui reproche de s'occuper de sciences auxiliaires de la géographie plutôt que de la géographie elle-même; on constate un défaut de préparation dans l'explication des auteurs. $»^{14}$ Puis on se fait à cette indépendance : « Esprit actif, ouvert, qui remue volontiers des idées personnelles sur les sciences auxiliaires de la géographie et qui montre du zèle et de l'ambition pour le développement et le fonctionnement de sa discipline» dit le rapport de 1898. Cette indépendance a été acquise par une démarche institutionnelle qui s'est faite « en douceur » : la transformation de la chaire en chaire de géographie, mais aussi par un investissement considérable d'Auerbach dans un nouveau champ intellectuel. En ceci, il participe à la vitalité d'une université (Birck, 1996; Febvre, 1954) et d'une faculté très dynamique, en histoire tout particulièrement (Livet, 1954), où l'interaction avec le milieu régional est de longue date érigée en principe, en raison du défi national (la Revanche) et de l'enjeu régional (l'esprit lotharingiste) qui se rencontrent dans la promotion de l'université nancéienne.

\section{Une chaire et un « Institut »}

18 Auerbach (professeur-adjoint en 1892) a formellement acquis son autonomie en obtenant que la chaire d'histoire et de géographie soit transformée en chaire spécialisée (décret du 1er janvier 1893, délibérations du Conseil de faculté le 30 novembre $\left.1892^{15}\right)$. Cette spécialisation va dans le sens des décisions ministérielles et de desiderata anciens de la faculté (Andrews, 1986). D’ailleurs, en décembre 1886, le Conseil de faculté maintenait par un vote unanime le libellé mixte de la chaire, en marquant sa vocation à permettre le nécessaire dédoublement de l'enseignement de l'histoire et à honorer la géographie : «à faire figurer à sa vraie place l'enseignement de la géographie, lequel est assez important pour appartenir à une chaire magistrale " ${ }^{16}$. Après la création en 1892 d'une chaire nouvelle consacrée à l'histoire de la France de l'Est, la qualité de la dotation dont bénéficient les historiens facilite la demande de transformation. Les attendus de cette demande se centrent bien sur des besoins spécifiques en géographie, estimant "que le développement croissant des études géographiques doit leur assurer dans l'enseignement supérieur une place indépendante et autonome », et qu'» il est de l'intérêt de la faculté des lettres de Nancy d'y organiser d'une façon complète et définitive [sic] l'enseignement de la géographie ». La délibération du Conseil de faculté montre toutefois des réticences (la transformation n'est acquise qu'à la majorité) et combien il est difficile de distendre les liens entre les deux disciplines ${ }^{17}$.

19 Le pas vers une science dotée d'une méthodologie spécifique, Auerbach l'a franchi dès 1888. Comme son collègue $\mathrm{C}$. Diehl le fait en faveur de l'archéologie, il réclame des crédits pour constituer des collections "de cartes, d'atlas, de reliefs en plâtre, de recueils sérieux d'information» (Annales de l'Est, 1888), et, dix ans après, pour « du matériel d'enseignement pratique, appareils photographiques, appareils de projection, etc. » Il commence à équiper un laboratoire comme ont tenu à en monter, sur le modèle des séminaires allemands ou sur celui des laboratoires scientifiques, M. Dubois en Sorbonne, Édouard Ardaillon à Lille, E. de Martonne à Rennes... Mais, personnage qui, selon le recteur Adam, « appartient plutôt, avec des qualités très réelles, à la famille des esprits critiques (il en faut), que des créateurs et des organisateurs ${ }^{18}$, Auerbach tarde à aménager l'» Institut de géographie » dont il parle à partir de 1904 : c'est seulement 
une fois devenu doyen (en 1908) et une fois conduit à aménager la faculté de lettres dans de nouveaux locaux qu'il s'y consacre de manière convaincante (en 1909) ${ }^{19}$.

Auerbach participe aussi activement à la réforme de l'agrégation d'histoire et géographie qu'E. Lavisse a engagée en obligeant les candidats à posséder un « Diplôme d'études supérieures " (décret du 28-7-1894). Avec ses trois collègues historiens (Diehl, Pariset et Pfister) il contribue au règlement local du diplôme et consacre une part de son enseignement à cette préparation. Sauf exception, les thèmes choisis portent sur le présent ou bien se branchent sur des questions d'actualité, et les étudiants sont censés recourir à un traitement cartographique et à la compilation de sources statistiques, démographiques et économiques ${ }^{20}$.

\section{Une science chorologique : la synthèse régionale}

21 Le combat d'Auerbach pour l'autonomie passe aussi par la défense et illustration d'une position épistémologique. Selon lui, par le point de vue "chorographique» ou « régional $»^{21}$, la « synthèse » que représente l'étude régionale autorise la géographie à se prévaloir du titre de science et lui confère son indépendance par rapport aux disciplines spécialisées. Cette position est argumentée diversement, la multiplicité des attitudes d'Auerbach attestant de son adhésion profonde à ce paradigme. Il se retrouve ici encore sur la ligne qui a rassemblé le groupe des Annales.

On peut repérer quatre ensembles d'interventions caractéristiques. La première est d'ordre programmatique. Exposé comme un manifeste dès le cours inaugural (1888), le parti de la géographie régionale est repris plus systématiquement en ouverture du Plateau lorrain (1893) et admis à nouveau, à la suite de Hettner et à l'exemple des travaux français, comme la meilleure définition de la géographie (1908). L'évolution des débats en Allemagne et l'orientation de la géomorphologie et de la géographie humaine le portent vers une appréciation plus fine des problèmes épistémologiques et des questions écologiques; il montre alors une certaine sensibilité à l'explication génétique (ou historique) des phénomènes qui ne le détourne cependant pas du parti chorologique qu'il a défendu d'emblée.

Ce parti lui permet aussi de distinguer entre géographes et non-géographes et entre savants et amateurs. C'est ainsi qu'il dénie à son collègue Bleicher le titre de "géographe professionnel » car, aussi bon connaisseur des Vosges et aussi polyvalent qu'il soit, il demeure un naturaliste qui ne s'intéresse aucunement aux "rapports » entre les phénomènes (compte rendu 1890 et 1901). Inversement, un voyageur, un journaliste, peut se révéler géographe s'il est capable de détecter des formes régionales : ainsi d'Ardouin-Dumazet (compte rendu 1901). Dans cette valorisation du «métier» de géographe, l'historien n'est pas oublié. Auerbach n'hésite pas à stigmatiser la perspective strictement lettrée de la culture historienne et l'étroitesse des conceptions politiques que cela recouvre, - la plus grande partie de l'humanité, vouée à une civilisation orale, étant dès lors exclue du champ de l'histoire universelle (1888).

24 C'est aussi par l'analyse détaillée de l'exemplarité des ouvrages de géographie moderne qu'il démontre la valeur de sa discipline. Le paradigme de la géographie est aux yeux d'Auerbach matérialisé par deux des ouvrages de Vidal de la Blache, l'Atlas historique et géographique Vidal-Lablache et le Tableau de la géographie de la France. Bien que ces ouvrages aient été présentés comme le manifeste et le chef d'œuvre de Vidal, rares 
sont les analystes qui ont mis en évidence leur nouveauté. Auerbach est de ceux-là, qui les élève tous deux à la hauteur de modèles. Dédaignant la paraphrase, il relève le motclé (la « caractéristique » d'une contrée), montre comment la présentation concrète de la feuille d'atlas, avec ses cartons et son commentaire succinct, réalise le projet chorologique en soumettant à la vue et à l'esprit «l'enchaînement et les rapports des faits». Non content de cet aperçu méthodologique, il démontre par l'exemple la capacité de l'approche géographique à dépasser l'histoire, même sur ses terrains de prédilection, notamment par les témoignages de la civilisation matérielle : ainsi «la première carte même de l'Atlas, celle de l'Égypte ancienne, fournit un modèle de la manière géographique: elle est semée d'indications dont les cartes historiques se passent volontiers : routes des caravanes, avec les citernes qui les jalonnent, carrières d'où ont été extraits les matériaux des monuments, mines de pierres précieuses qui sollicitent le commerce à travers le désert » (compte rendu, 1894). L'analyse de la partie proprement géographique est l'occasion de marquer la spécificité de la méthode synthétique, de telle sorte que cet atlas lui paraît être littéralement "l'expression figurée de la science géographique » (ibid.). Une pareille acuité anime son commentaire du Tableau (1904).

Enfin, introducteur de la Länderkunde à la française auprès de ses collègues allemands, Auerbach (1899) est lui-même reconnu parmi les spécialistes de ce genre par Otto Schlüter (1910). Pionnier dans l'affirmation du programme de recherche chorologique, Auerbach (1891-1892 et 1893) applique effectivement la méthode préconisée en découpant dans l'Est de la France une «individualité géographique lorraine » et en la subdivisant en ses "cellules» élémentaires, les pays. Cette opération de découpage repose sur la prise en compte d'une unité physionomique d'ensemble, d'ordre topographique et géologique, qui coïncide grosso modo, pour le "plateau lorrain » (1893) ou la "terre lorraine» (1909), avec les assises jurassiques et triasiques. Quant aux multiples parties de l'ensemble, Auerbach les détecte essentiellement par l'examen de la carte géologique à grande échelle, la description détaillant, au prix d'une très abondante documentation, la vie économique et démographique contemporaine. Au total, les divisions adoptées par Auerbach restent fort dépendantes de la géologie (Bonnefont, 1993b).

\section{Géographie régionale et régionalisme}

En affichant une identité lorraine distincte des entités historiques et administratives, Auerbach participe à la critique que les géographes adressent depuis les années soixante-dix à des divisions territoriales qu'ils jugent arbitraires, et il contribue à leur combat en faveur de "régions naturelles». Il rencontre comme eux le mouvement régionaliste qui s'épanouit en France au tournant du XIX-XXe siècle. M.-V. OzoufMarignier (1998) a montré que «le fait d'établir leur objet, l'étude des divisions naturelles, par opposition avec les divisions administratives en butte alors à la critique de l'opinion, [a placé] les géographes en experts dans le débat public ». Quelle position Auerbach adopte-t-il dans cette conjoncture où se côtoient donc une dynamique disciplinaire et un mouvement social et politique diffus, dans lequel le milieu universitaire nancéien a été pris, comme l'attestent la sensibilité de la faculté des lettres au thème régionaliste et, concrètement, la création d'une chaire d'histoire régionale ${ }^{22}$ ? 
27 On a déjà souligné la précocité de son intérêt pour l'approche régionale et pour la géographie de la Lorraine. Mais un aspect spécifique des interventions d'Auerbach parmi ses confrères des Annales tient à l'attention qu'il accorde à la question de la décentralisation. Elle s'épanouit au tournant du siècle. En témoignent les cours sur « les pays de France » et sur " les provinces françaises » qu'il professe en licence au début des années 1900. Plus suggestif apparaît le contenu de diplômes de géographie soutenus à la même époque, qui portent sur la « colonisation intérieure de la France au cours de ce siècle » (1899), sur «la décentralisation administrative d'après les données géographiques» (1902) ou sur la départementalisation en Lorraine (1905). Dans son rapport sur le diplôme relatif à la décentralisation en Lorraine, le jury suggère de donner une orientation économique à la recherche d'un nouveau découpage: «Bien que le candidat ait étudié de près les conditions physiques et la vie de la province et rassemblé des matériaux statistiques de toute nature, illustré de graphiques et de cartogrammes, il n'a pas abouti à une conclusion ferme; il s'est borné à rejeter les divisions géologiques ou topographiques comme bases de circonscriptions nouvelles ; il n'a pas essayé de délimiter d'autres groupements homogènes d'après l'industrie ou les intérêts économiques; il n'a pas davantage recommandé le maintien des cadres administratifs. $»^{23}$

Dès 1901, Auerbach a pris parti pour un programme de décentralisation et a proposé aux Sociétés de géographie de discuter de projets de regroupements reposant sur de «libres associations d'intérêts ${ }^{24}$. Cette implication dans l'humeur régionaliste et l'orientation économique de la critique des divisions territoriales se confirment par les propos tenus par Auerbach lors de conférences à l'Université populaire de Nancy, telle «la décentralisation du point de vue géographique » (janvier 1902). Selon le compte rendu, il reprend une argumentation classique, rappelant au passage le Programme de Nancy, critiquant les solutions du géographe Pierre Foncin, et suggérant in fine un découpage fondé sur l'organisation économique autour d'une ville: «M. Auerbach pense qu'il serait peut-être plus rationnel de grouper autour d'un centre tous les habitants qui vivent plus spécialement de l'exploitation d'un produit naturel ou d'une industrie plus particulièrement prépondérante. C'est ainsi que Nancy pourrait devenir le centre administratif et commercial de l'industrie du fer, Lyon celui de la soie, StÉtienne celui de la houille, etc. $»^{25}$

Auerbach envisage par là une division moderne à larges mailles. Il a en effet compris dans les articles que L. Gallois a consacrés à la région lyonnaise au début des années quatre-vingt-dix le rôle d'une métropole industrielle dans l'animation d'une "grande province moderne ». Il y fait allusion dans son compte rendu des travaux français (1899) et y revient à propos de la thèse secondaire d'E. de Martonne : "Grandes ou petites, ces provinces modernes qui émaillent la surface de l'Europe occidentale se concrètent [sic], par la solidarité et par la prédominance des phénomènes économiques et sociaux, en régions naturelles, elles aussi, bien que les linéaments physiques y soient, si l'on peut dire, estompés. C'est à les déterminer que beaucoup d'auteurs se sont ingéniés » (1903, p. 362, c'est nous qui soulignons). Il ne semble pas qu'il ait perçu l'effet structurateur de la métropole moderne avec la généralité qui lui sera assignée une décennie plus tard autour des travaux que Vidal de la Blache consacre alors à la région moderne (Ozouf-Marignier, Robic, 1995). Il ne l'applique pas non plus à l'analyse de la Lorraine, la place qu'il accorde à Nancy étant très faible dans chacune de ses 
publications ${ }^{26}$, alors qu'il étudie toutes les petites villes, souvent en suivant le fil des fleuves...

Allant au-delà de la lettre de l'ouvrage, l'analyse qu'Auerbach accorde au Tableau de la géographie de la France implique ouvertement Vidal dans la question du régionalisme. Avec précaution mais fermeté, il va même jusqu'à postuler une adéquation entre le travail du géographe et le point de vue décentralisateur : « $\mathrm{M}$. Vidal de la Blache est trop géographe pour célébrer la centralisation. (...) Ce qui console M. Vidal de la Blache, si nous interprétons bien sa pensée, c'est que la centralisation n'est peut-être pas le terme fatal du développement de la France (...) Le procès n'est pas tranché ; M. Vidal de la Blache se garde de prononcer ou même de préjuger une sentence. (...) Il nous facilite l'intelligence du passé, mais aussi bien celle de l'histoire de demain ; l'on trouve ici en substance tous les arguments d'un programme de décentralisation, d'un remaniement des cadres politiques, d'un plus judicieux emploi des forces vives du pays; et ces considérations nous ramènent à la définition du vieux maître Strabon (...); la Géographie répond surtout aux besoins de la vie politique » (1904, p. 899, c'est nous qui soulignons). L'origine de cette interprétation, c'est bien entendu la critique que fait Vidal lui-même des méfaits de la centralisation monarchique. Analysant le Tableau à la lumière des grands articles de Vidal, Auerbach souligne avec lui le rôle joué par les moyens de communication dans la géographie humaine. De même, il montre la signification des villes pour Vidal, "des pôles d'énergie vitale, des points de cristallisation autour desquels les éléments des formations politiques se sont conglomérés» (1904, p. 898); mais les exemples qu'il rappelle sont des capitales anciennes, plutôt que ces métropoles modernes, telle Lyon, que Vidal a célébrées dans le Tableau. Peut-être la sensibilité d'Auerbach le porte-t-elle à valoriser la circulation plutôt que les villes organisatrices, une circulation qui est effectivement l'une de ses obsessions.

Malgré ces hésitations d'Auerbach, son inspiration décentralisatrice est bien ancrée, et il l'a partagée avec ses étudiants. S'il n'a pas eu beaucoup d'élèves, l'un d'eux, Émile Chantriot, a adressé dans sa thèse un hommage à son "maitre » Auerbach et exprimé dans le même temps sa foi régionaliste. Étudiant de la faculté des lettres de Nancy, boursier d'agrégation à Nancy en 1887-1888, agrégé d'histoire et géographie en 1892, É. Chantriot soutient en 1905 à Paris une " étude de géographie régionale » consacrée à la Champagne. Alors qu'à l'époque les monographies régionales sur la France, que la thèse d'Albert Demangeon a inaugurées la même année, ne comportent ni justification scientifique initiale ni position politique, Chantriot situe sa recherche dans un mouvement régionaliste dont il admet partager les objectifs : célébrer les «avantages qu'il y aurait à briser les entraves de la centralisation et à développer des libertés locales seules capables de stimuler l'activité des foyers de la vie régionale et de l'initiative individuelle»(p. VI). Il estime que son travail relève de l'expertise scientifique du géographe, et il réserve à la conclusion l'examen des principes des divisions spatiales requises par la modernité. Aussi préconise-t-il une organisation moulée sur les réseaux de circulation et sur les pôles économiques, et propose-t-il une délimitation de la Champagne qui fait fi des traditions et des divisions physiques...

On observe donc chez ces deux géographes travaillant en province et sensibilisés à la question régionale dans le milieu nancéien une même tension vers l'expertise. En même temps ils affrontent (la conclusion de Chantriot le montre bien) la difficulté qu'ils ont à concilier l'objet de géographie régionale pure (les régions naturelles de 
diverses échelles) et celui qui est au cœur du débat civique, la région moderne. Cette contradiction entre projet épistémologique et pertinence sociale ne leur est pas réservée. L. Gallois l'observera au terme de son étude sur Régions naturelles et noms de pays, mais pour conclure, en gardien de la loi, à la valeur intrinsèque de la région naturelle. À l'inverse, la démarche d'Auerbach se libère d'une conception strictement naturaliste et surtout de l'entreprise rationalisante et légitimatrice de Gallois : science morale à fondement naturel, comme il l'entendait dans son cours inaugural en 1887, science anthropocentrée, la géographie appartiendrait avant tout à la «science sociale ", comme il l'estime en 1903, ou même à la "science politique ", comme il le pose en 1904 en se mettant sous le patronage de Strabon. S'il est légitime de penser que cette orientation régionaliste fait écho à la vivacité des revendications politiques, culturelles et économiques fédérées dans le mouvement régionaliste, si Auerbach se montre perméable aux thèmes décentralisateurs et particularistes qui agitent l'opinion en Lorraine, on peut estimer au vu de ses autres engagements qu'il est animé d'une conviction propre de citoyen.

\section{Un géographe démocrate...}

Du jeune professeur qui s'attire les foudres des autorités parce qu'il affiche sur un mode léger ses opinions antimonarchiques et antipapistes, valorisant quand il le peut les mérites de la Révolution, jusqu'à son long engagement dans l'Université populaire de Nancy, Auerbach présente durant sa carrière une attitude anticonformiste ou du moins indépendante. Son dossier personnel de fonctionnaire suggère un irrespect envers l'autorité et une aspiration démocratique - d'aucuns l'estiment démagogique qu'Auerbach manifeste dans l'impertinence face à l'Inspection, dans une certaine familiarité avec les élèves ou dans son refus de noter ses collègues (c'est-à-dire de renseigner le recteur d'académie, en tant que doyen de faculté). L'un des rares témoignages que nous connaissons sur le personnage signale aussi comment il savait à la fois honorer et désacraliser la chaire universitaire: «M. Bertrand Auerbach professait la géographie avec beaucoup de vie; il a eu le mérite de vouloir, un des premiers, intéresser ses élèves à ce qu'ils avaient sous les yeux, aimant mieux les courber sur le sol lorrain, qu'ils foulaient, que sur la carte du Turkestan, petit homme vif et alerte qui, bien avant qu'on eût inventé la géographie humaine, la professait; c'est lui qui installait dans sa propre chaire l'élève chargé de la leçon, et, voyez, moi qui ne devais pas être professeur, cela me plaisait beaucoup, peut-être par le pressentiment que j'en resterais là » (Madelin, 1934).

On a vu Auerbach lancer des piques à l'historien pour cause de mépris à l'égard des peuples sans écriture ; il applaudit à des entreprises coloniales privilégiant la justice ou la visée humanitaire sur l'objectif mercantile : il manifeste une ouverture à la vie de la Cité qui explique que, géographe moderne (qui préfère toutefois l'érudition livresque à la fréquentation du terrain), Auerbach ne s'est pas enfermé dans sa discipline. Peutêtre même a-t-il versé dans la géographie parce qu'elle lui donnait une prise sur le contemporain: déjà, lors d'une leçon présentée en histoire ancienne, Fustel de Coulanges remarquait que ce jeune élève " pens[ait] trop au présent $»^{27}$ !

Comment s'enfermer en une tour d'ivoire à Nancy, alors que la ville et son Université sont chargées d'une tâche symbolique face à l'Allemagne triomphante? Patriote, Auerbach ne manque jamais de déplorer l'amputation de l'Est de la France, en 1893, où 
il inclut dans son livre l'étude de la Lorraine devenue allemande, comme en 1909, où il rappelle que la Lorraine reste « la "Marche de l'Est", sacrée par la mutilation subie » (p. 59). Il compare la partie française et la partie devenue allemande, cherchant à dégager dans les phénomènes démographiques les mérites de sa patrie. Il témoigne, il confronte, sans déployer jamais de nationalisme agressif. En revanche il est un observateur attentif et critique de l'Allemagne, de son dynamisme, de son équipement commercial, de ses voies navigables et de ses poussées expansionnistes (aux Annales de géographie, dans la Revue générale des sciences, dans la Revue politique et parlementaire...).

Enfin son engagement des années 1900 dans le mouvement des Universités populaires montre qu'Auerbach se laisse interpeller par l'actualité comme savant et comme homme de convictions. En conférencier polyvalent et en militant, il participe à ce brassage rationaliste et démocrate entre intellectuels et milieux ouvriers, qui dérange fortement une ville conservatrice comme Nancy ${ }^{28}$. De 1902 à 1908, Auerbach y traite de sujets très divers, les uns relevant de sa spécialité, telles les questions de décentralisation ou l'analyse du conflit Russie-Japon (1904), d'autres paraissant plus idiosyncrasiques telles une causerie sur le « compagnonnage » ou une conférence sur le "prophétisme", d'autres encore s'intéressant à l'actualité éditoriale, sociale ou politique: conférence sur le "rôle social du catholicisme d'après Brunetière ", conférence sur "le Prolétariat russe" qui annonce une "révolution sociale", présentation de "Malthus et le malthusianisme », conférence sur « ce qu'on entend par unité morale du pays "... Auerbach ne se contente pas d'être l'un de ces savants qui viennent de temps à autre dispenser leur savoir à un public populaire. Il est aussi membre du «Comité » élu et préside nombre de réunions.

\section{Du compagnonnage à l'éclipse ?}

Pendant trois décennies, Auerbach participe à l'équipe organisée autour des Annales de géographie. Il y publie en effet dès le volume 2 et régulièrement, au rythme d'un article par an jusqu'en 1907. Surtout sa collaboration suivie à la Bibliographie annuelle lancée par la revue marque sa collaboration à ce « collectif » intellectuel : de 1896 à l'aprèsguerre il se consacre aux rubriques "Alsace-Lorraine " et "Allemagne ${ }^{29}$ avec une constance étonnante et cette énergie de gros travailleur qui lui est reconnue dès l'École normale. Il rédige environ 600 comptes rendus, dont une cinquantaine sur l'AlsaceLorraine, soit une moyenne annuelle de 34 notices $^{30}$. Ses dernières contributions datent de 1922, ce retrait semblant lié au renouvellement de la Bibliographie à partir de 1923, avec l'introduction de jeunes géographes français et de collaborations étrangères ${ }^{31}$. Auerbach a aussi été admis à traiter aux Annales de la thèse secondaire d'E. de Martonne. Celle-ci tombe dans l'un de ses champs de compétences, la cartographie démographique. S'ajoutant aux affinités épistémologiques évoquées ci-dessus, et en dépit du ton ironique qu'il adopte souvent, ceci témoigne d'une certaine confiance de l'équipe dirigeante des Annales.

38 Peut-on cependant déceler une rupture entre Auerbach et ses collègues géographes à la fin de la guerre, qui se serait traduite par une " condamnation sans appel » prononcée par Vidal dans La France de l'Est, à la suite de divergences sur l'avenir de l'Alsace et de la Lorraine (Bonnefont, 1993a, p. 87-88) ? Plus généralement, peut-on penser que, en 1917, «les positions d'Auerbach, favorable au germanisme et au maintien de la 
souveraineté autrichienne sur les pays slaves et hongrois lui aliènent la communauté géographique française" (Bonnefont, 1993b, p. 261)? La situation nous paraît plus complexe, et nous ne partageons pas l'idée que Vidal l'ait « exécuté ».

On peut relever des lacunes dans les signes de reconnaissance que les «patrons » de la géographie française lui auraient envoyés. La plus apparente concerne la Géographie Universelle, à laquelle Auerbach n'a pas participé. Si la collection paraît seulement durant l'entre-deux-guerres, sa publication devait démarrer vers 1912-1913: or Auerbach ne figure pas dans la liste dressée par Vidal de la Blache en 1908. C'est une équipe de jeunes qui est rassemblée (les trois quarts ayant entre 30 et 40 ans en 1908 et des auteurs comme L. Gallois, qui entrait dans la cinquantaine, et p. Camena d'Almeida, faisant figure de vétérans). L'exclusion d'Auerbach tient probablement au fait qu'il n'a pas démontré, aux yeux de Vidal, ses capacités à produire ce morceau de la géographie du monde qui est attendu des futurs collaborateurs ${ }^{32}$. En outre, pour ce spécialiste de l'Allemagne et de l'Autriche-Hongrie, la concurrence est très défavorable puisqu'il s'oppose, en la personne d'E. de Martonne, au jeune géographe qui peut prétendre à l'Europe centrale en vertu de la double excellence qu'il a démontrée dans ses thèses de lettres et de sciences.

Auerbach est aussi en retrait par rapport à l'activisme que les géographes vidaliens ont déployé pendant la guerre pour le renseignement militaire et pour la préparation des traités (Ter Minassian, 1997). Il n'en est pas complètement absent puisqu'il collabore dès 1915 à la Commission de géographie chargée de rédiger des notices pour l'armée ${ }^{33}$ et qu'il bénéficie d'un congé pour servir comme expert auprès de la Conférence de la Paix de janvier à mars 1919. Au vu de ses compétences en matière politique et ethnographique sur l'Europe centrale, son rôle reste très mineur. Nous y voyons quatre facteurs : d'abord son éloignement de Paris alors que le travail sur les notices se faisait dans l'urgence et que les collaborateurs du "Comité d'études» réuni par Charles Benoist en 1917 avaient été recrutés dans ses réseaux parisiens; son inexpérience en matière de pratique cartographique - où de Martonne a fait merveille - ; à nouveau la concurrence écrasante avec celui-ci sur l'Europe centrale. Le quatrième facteur serait d'ordre politique. Si l'on en juge par les positions qu'il a exprimées dans ses travaux scientifiques et dans ses articles politiques, Auerbach ne devait pas soutenir une volonté aussi délibérée d'écrasement de l'Allemagne ni de démantèlement de l'Autriche-Hongrie que celle qui prévalait parmi les négociateurs français: dans ces conditions, il est fort possible qu'il ait été marginalisé, là où de Martonne, parfaitement en phase avec la ligne française officielle, pouvait s'imposer comme expert.

Cette réserve quant au démantèlement de la monarchie austro-hongroise, Auerbach l'a justifiée en introduisant son livre sur les Races et nationalités de l'Autriche-Hongrie (1898 et 1917), où il dit son aspiration à une structure d'État fédératif qui accorde expression et indépendance aux peuples coexistant dans l'empire. Son livre sur la politique de l'Autriche et la Hongrie pendant la guerre (1925) analyse les conditions du parfait échec de la ligne libérale qu'il avait espérée de leur part. $\mathrm{Si}$, dans le système politique austro-hongrois, il admettait jusqu'à un certain point la prééminence de la civilisation allemande (le terme valant ici plus que celui, particulariste, de "culture ", et sachant qu'il distingue volontiers le Prussien), ses publications ne peuvent laisser de doute sur l'âpreté de sa critique à l'égard des «Allemands autrichiens » $(1910,1917$ et 1917), et plus généralement sur sa condamnation du pangermanisme $(1908,1913)$. Dans des textes de genre scientifique ou journalistique, il condamne l'impérialisme sous- 
jacent à l'expansion du Deutschtum parce que le pangermanisme est négateur de l'altérité et par là destructeur. Aussi ne nous parait-il pas vraisemblable que Vidal de la Blache, lui aussi ambivalent à l'égard du monde germanique, l'ait visé. Dans la petite phrase où il évoque « l'écrivain pangermaniste Auerbach » à propos de l'état d'esprit des Alsaciens après la Révolution et les guerres napoléoniennes Vidal de la Blache désigne très probablement Berthold Auerbach (1812-1882), un auteur allemand extrêmement célèbre à la fin du XIXe siècle, connu notamment pour ses "Récits villageois de la Forêt-Noire » (Schwarzwälde Dorfgeschichten).

Du cours inaugural de 1887 aux derniers travaux du professeur Auerbach, l'itinéraire du géographe trace donc une longue courbe, de la critique d'une École spéciale de géographie jusqu'à la participation à l'essor de l'École française de la géographie. Ce compagnonnage est suivi d'une prise de distance réciproque : le pionnier qui a cultivé la géographie loin de la Sorbonne se voit dédaigné par la jeune génération tandis qu'il se replonge de son côté dans l'histoire diplomatique qui fut sa première passion et qu'il se plaît à analyser les turbulences de la politique européenne du temps présent.

Les normaliens historiens des promotions 1876-1881 qui se sont convertis à la géographie ont eu des parcours contrastés : à côté de Dubois le géographe "colonial ", l'hétérodoxe, de Dupuy, le pédagogue devenu comme Surveillant général le pilier de l'École normale, de Gallois, le «lieutenant» fidèle de Vidal, Auerbach fait figure d'éclaireur vite oublié. Sans doute n'a-t-il pris part à l'affirmation de la géographie moderne française qu'avec une certaine réserve, sans mettre en œuvre lui-même de manière approfondie ses partis intellectuels sur la synthèse régionale ou sur le " pays ", en restant un érudit critique plutôt qu'un homme de terrain, en suggérant souvent la dette intellectuelle des géographes français à l'égard de la science allemande... La position nancéienne d'Auerbach a produit une interaction originale entre la conception chorologique de la discipline et les intérêts régionalistes, qui ne le conduit pas luimême à remettre en cause les présupposés de sa démarche, mais qui l'entraine à définir plus fermement la géographie comme science sociale. Elle lui a permis aussi, depuis ce poste avancé de la France, d'exercer une analyse critique du germanisme, jusqu'à se tourner vers une forme de journalisme politique où il a réinvesti un sens historique formé initialement aux questions diplomatiques, et son savoir-faire de géographe moderne.

\section{BIBLIOGRAPHIE}

Andrews H.F. (1986). - « Les premiers cours de géographie de Paul Vidal de la Blache à Nancy (1873-1877) », Annales de géographie, p. 341-361.

Birck F. (1996). - « Le développement de l'enseignement supérieur à Nancy, une volonté politique », in : Grossetti Michel, Grelon André (dir.), Programme « Villes et institutions scientifiques ». Rapport final, PIR-Villes CNRS, 360 p. dact., p. 150-166. 
Bonnefont J.-C. (1993a). - « Bertrand Auerbach (1856-1942). Un géographe nancéien subtil et mal aimé », Nancy, Mémoire de l'Académie de Stanislas, Séance du 19 mars 1993, p. 249-261.

Bonnefont J.-C. (1993b). - « La Lorraine dans l'œuvre de Paul Vidal de la Blache », in : Claval Paul (dir.), Autour de Vidal de la Blache. La formation de l'école française de géographie, Paris, CNRS Éditions, p. 81-88.

Broc N. (1974a). - « Bertrand Auerbach (1856-1942). Un pionnier de la géographie en Lorraine », Revue géographique de l'Est, 3-4, p. 411-415.

Broc N. (1974b). - «L'établissement de la géographie en France : diffusion, institutions, projets (1870-1890) », Annales de géographie, p. 545-565.

Broc N. (1977). - « La géographie française face à la science allemande (1870-1914)», Annales de géographie, p. 71-94.

Capel H. (1982). - « Institutionalization of geography and strategies of change », in : Stoddart D.R. (ed.), Geography, ideology and social concern, Oxford, Basil Blackwell, p. 37-69.

Chantriot É. (1906). - La Champagne. Étude de géographie régionale, Paris/Nancy, Berger-Levrault.

Dubois M. (1888). - «L'avenir de l'enseignement géographique », Revue internationale de l'enseignement, XV, p. 449-477.

Febvre L. (1954). - «L'Université de Nancy de 1890 à 1900. Souvenirs et leçons », Annales de l'Est, p. 175-183.

Karady V. (1973). - « L'expansion universitaire et l'évolution des inégalités devant la carrière d'enseignant au début de la troisième République ", Revue française de sociologie, p. 443-470.

Livet G. (1954). — « La Faculté des Lettres de l'Université de Nancy. Son enseignement et ses étudiants de 1871 à 1914 », Annales de l'Est, p. 211-239.

Madelin L. (1934). - «Christian Pfister », Annales de l'Est, p. 129-152.

Meynier A. (1969). - Histoire de la pensée géographique en France (1872-1969), Paris, PUF, 224 p.

Ozouf-Marignier M.-V. (1998). - « Les géographes et le découpage administratif de la France », in : Pertué M. (dir.), L'administration territoriale de la France (1750-1940), Orléans, Presses universitaires d'Orléans, p. 3-14.

Ozouf-Marignier M.-V., Robic M.-C. (1995). - « La France au seuil des temps nouveaux. Paul Vidal de la Blache et la régionalisation », L’Information géographique, p. 46-56.

Ozouf-Marignier M.-V., Robic M.-C. (1998). - « Un tableau à vif. la réception du Tableau de la géographie de la France de p. Vidal de la Blache », in : Robic M.-C. (dir.), Dans le labyrinthe des formes. L'identité française d'après le Tableau de la géographie de la France de P. Vidal de la Blache, Paris, Comité des travaux historiques et scientifiques (à paraître).

Palsky G. (1996). - Des chiffres et des cartes. Naissance et développement de la cartographie française au XIXe siècle, Paris, Comité des travaux historiques et scientifiques, 331 p. (chapitre 5)

Pinchemel p., Robic M.-C., Tissier J.-L. (dir.), (1984). - Deux siècles de géographie française. Choix de textes, Paris, Comité des travaux historiques et scientifiques, $380 \mathrm{p}$.

Robic M.-C. (1993). - « La creacion de los Annales de Géographie, 1891. Estrategia universitaria y geografia humana », Documents d'Analisi Geografica, 22, p. 47-64.

Schlüter O. (1910). — « Über einige neuere Werke zur französischen Landeskunde », Geographische Zeitschrift, XVI, p. 605-617 et 665-689. 
Soubeyran O. (1997). - Imaginaire, science et discipline, Paris, Montréal, L'Harmattan, 482 p.

Ter Minassian T. (1997). - « Les géographes français et la délimitation des frontières balkaniques à la Conférence de la paix en 1919 ", Revue d'histoire moderne et contemporaine, 44 (2), p. 252-286.

Vidal de la Blache p. (1917). - La France de l'Est (Lorraine-Alsace), Paris, F. Alcan, 285 p. (Réédition 1994, Paris, Éditions de La Découverte, présentée par Yves Lacoste).

\section{ANNEXES}

\section{- Bertrand Auerbach, Bibliographie personnelle}

Abréviations des noms de revues : AE : Annales de l'Est ; AESP : Annales de l'École des Sciences politiques; AG : Annales de Géographie ; AGG : Archives de la Grande Guerre ; GZ : Geographische Zeitschrift ; JS : Journal des Savants ; RG : Revue de géographie ; RGP : Revue générale des Sciences ; RP : Revue de Paris ; RS : Revue scientifique ; RPP : Revue politique et parlementaire; $\mathrm{S}$ : Scientia.

1887, La diplomatie française et la cour de Saxe (1648-1680), Paris, Hachette, XXIV- 492 p. (thèse principale).

1887, Quid sibi voluerit Strabo rerum geographicum libros componendo?, Nancy, $94 \mathrm{p}$. (thèse latine).

1888 et 1889 , « Buffon géographe », RG, 1888, II, p. 400-412 et 1889, I, p. 16-24, 114-123 et $175-185$.

1888, « Caractère et tendance de la science géographique », AE, II, p. 44-66.

1889, « La question d'Alsace à la Diète de Ratisbonne », AE, III, p. 309-336.

1890-1892, « La Lorraine, essai de chorographie »; RG 1890, I, p. 175-185 ; 1890, II, p. 9-21, 169-176, 253-270 ; 1891, II, p. 101-118, 412-433.

1892-1993, « Étude sur le régime et la navigation du Rhin », AG, II, p. 212-238.

1893, « Distribution et rôle géographique des routes nationales », RS, 27 mai 1893.

1893, Le plateau lorrain. Essai de géographie régionale, Paris, Nancy, Berger-Levrault, 359 p.

1894, « Note sur la démographie de l'Alsace-Lorraine comparée à celle de la France et de l'Allemagne », AE, p. 222-232.

1894, « La colonisation des tourbières du Nord-Ouest de l'Allemagne », AG, III, p. 227-241.

1894, «Les résultats de la dernière expédition d'Emin-Pacha. Relief, géologie, hydrographie ", AG, III, p. 76-85.

1895, « Un problème de colonisation intérieure : la germanisation de la Pologne prussienne », AESP, 15 mars.

1895-1896, «La répartition géographique de la population sur le sol allemand », AG, V, p. 59-71 et 469-482.

1897, « Le régime de l’Oder », AG, VI, p. 313-327. 
1898, « La carte de Lorraine sous le duc Charles III (Gérard Mercator, Hans van Schille, Thierry Allix) », RG, XIII, p. 321-333.

1898, « Le Danube austro-allemand et les projets de jonction de ce fleuve avec le Main, l'Elbe et l'Oder », RGS, IX, p. 749-755, 769-776.

1898, Les races et les nationalités en Autriche-Hongrie, Paris, F. Alcan, 333 p. (2e édition revue, 1917).

1899, "Le commerce maritime et la marine marchande de l'Allemagne », AG, p. 357-364.

1899, « Les chemins de fer de l'Indoustan », RGS, X, p. 730-741.

1899, «L'Orléanais », in : Dictionnaire géographique et administratif de Joanne, Paris, Hachette.

1899, « Über französiche Länderkunde », GZ, 5 (11), p. 580-587 et 618-630.

1901, « Les travaux géographiques de G. Bleicher », AE, XV, p. 592-609.

1901, « Les dunes d'Allemagne », AG, p. 272-273.

1902, « La politique prussienne et les voies navigables », La Grande Revue, XXIII, 1er juillet, p. 65-84.

1902, "Le canal du Nord-Est », in : Congrès national des Sociétés françaises de géographie, 22e session, Nancy 1-5 août 1901. Comptes rendus publiés par la Société de géographie de l'Est, Nancy, Berger-Levrault, 1902, p. 140-160.

1902, « Le canal du Nord-Est », RGS, 13, p. 140-151.

1902, « Le régime de l’Elbe », AG, p. 54-67 et 134-143.

1903, « Le régime de la Vistule », AG, p. 214-234.

1903, « La distribution de la population en Valachie », AG, p. 360-362.

1903, « Le Tableau de la géographie de la France de M. Vidal de la Blache », RGS, 14, p. 895-900.

1904, « Le régime de la Weser », AG, p. 138-144 et 257-265.

1905, « La politique coloniale aux débuts de la Restauration », RPP, 46, p. 333-352.

1905, « La population de l'Empire allemand d'après le recensement de 1900. Phénomènes démographiques et industriels. Statistique confessionnelle et linguistique », AG, p. 118-125.

1907, « La Moselle et la Sarre dans le programme de navigation intérieure de l'Allemagne », RPP, 54, p. 352-364.

1907, « Le régime de la Moselle, d'après un ouvrage récent », AG, p. 23-30.

1907, « Le peuplement national en Algérie 1871-1906 », RGS, 18, p. 307-311.

1908, « L'évolution des conceptions et de la méthode en géographie », JS, p. 309-319.

1908, « La germanisation de la Pologne prussienne. La Loi d'expropriation », RPP, 57, p. 109-125.

1908, « La formation de l'État anglo-égyptien », JS, p. 643-655. 
1909, « La terre lorraine » et « Les voies de communication en Lorraine », in : Nancy et Lorraine, Société industrielle de l'Est, Paris, Dunod et Pinat, 244 p., p. 59-73 et p. 125-135.

1910, « La valeur sociale des Allemands dans la Monarchie autrichienne », RPP, 64, p. 236-251.

1912, La France et le Saint-Empire romain germanique depuis la Paix de Westphalie jusqu'à la Révolution française, Paris, Champion, LXXIII-485 p.

1911, « Le problème indien devant l'opinion publique anglaise », RPP, 68, p. 469-485.

1912, "Introduction et notes ", in : Recueil des instructions données aux ambassadeurs et Ministres de France depuis les traités de Westphalie jusqu'à la Révolution française. XVIII. Diète germanique, Paris, F. Alcan, XCVIII-400 p.

1912, « Préface », in : Joly H., Géographie physique de la Lorraine et de ses enveloppes, Nancy, Albert Barbier, p. I-XII.

1913, « Le pangermanisme. Son organisation, son action », RPP, 78, p. 14-35.

1913, « L'industrie du coton en Alsace : les conditions géographiques d'une grande industrie », AG, p. 94-98.

1917, “"L'Autriche nouvelle” des Allemands autrichiens », RP, 15 oct., p. 843-891.

1917, Les races et les nationalités en Autriche-Hongrie, Paris, F. Alcan, 2e édition revue, $492 \mathrm{p}$.

1921, « La crise de l'Alliance austro-allemande sous le ministère du Comte Czernin (1916-1917) », AGG, oct-nov, déc.

1921, « La question de l'Union de l'Autriche allemande à l'Allemagne (Le mouvement de rattachement depuis la fin de la guerre jusqu'au traité de Versailles) », S, 30 (2), p.

201-212.

1922, La dictature du prolétariat en Hongrie (22 mars-31 juillet 1919), Paris, Chiron, 109 p.

1923, « Régions naturelles et pays », in : Champagne, Ardenne, Vallée de la Meuse, Paris, Hachette (Guides bleus), p. XVI-XXXIV.

1924, « Préface », in : Albert Collignon. Reliquiae, AE, p. V-XIII.

1925, L'Autriche et la Hongrie pendant la guerre (août 1914-novembre 1918), Paris, F. Alcan, $627 \mathrm{p}$.

1927, Le rattachement de l'Autriche à l'Allemagne, Nancy, Paris, Berger-Levrault, XI+191 p.

1928, « Aperçu géographique », in : Vosges, Champagne (sud), Lorraine, Alsace, Paris, Hachette (Guides bleus), p. XVIII-XLVIII (avec Pierre Deffontaines) (version révisée, 1934, p. XVII-XXXVII).

\section{Traductions}

1883, Curtius E., Histoire grecque. 4. La domination de Sparte en Grèce, Paris (sous la direction de M. Bouché-Leclercq).

1929, La politique extérieure de l'Allemagne 1870-1914. Documents officiels publiés par le Ministère allemand des Affaires étrangères. t. VII 25 mars 1890-6 mai 1891, Paris (Publication de la Société d'histoire de la Guerre), Alfred Costes, 345 p. 


\section{Comptes rendus dans les Annales de l'Est (sélection)}

1890, « G. Bleicher. Les Vosges, le sol et les habitants », Paris, Baillère et fils, 1890..., p.

316-319.

1894, « Atlas général Vidal-Lablache.... », p. 609-612.

1901, « Ardouin-Dumazet, Voyage en France, 21e série, Haute-Champagne, Basse-

Lorraine, 22e série : Plateau lorrain et Vosges, 23e série : Plaine comtoise et Jura, 1900

et $1901 \ldots$ », p. 319-322.

- Comptes rendus dans la Bibliographie des Annales de géographie (1896-1922) : environ 600 .

\section{NOTES}

1. La référence aux travaux de $B$. Auerbach est indiquée par la date de publication entre parenthèses. La liste de ses travaux figure dans l'annexe: Bertrand Auerbach. Bibliographie personnelle. Les sources d'archives sont repérées ci-dessous par les cotes des Archives nationales (AN) où nous citons notamment son dossier personnel (carton F17 23811) et celles des Archives départementales de Meurthe-et-Moselle (AD).

2. Auerbach n'a eu de nécrologie ni dans les Annales de géographie ni dans la Bibliographie géographique internationale ni dans l'Annuaire de l'École normale.

3. Sur la création de cette chaire voir Andrews, 1986.

4. A. Debidour, A. Rambaud, J. Zeller, T. Homolle, C. Pfister.

5. Outre les remarques de ses collègues sur la verve d'Auerbach, voir les critiques que lui adressent plusieurs professeurs à l'École normale (AN : 61AJ184), le conflit avec le proviseur du lycée de Laval et l'inspecteur pour "fâcheux écarts dans ses cours » et «érudition malsaine " (AN : $\mathrm{F}^{17}$ 23811) et, avec une tonalité raciste, la charge de l'inspecteur général Perrens qui rapporte sur la soutenance de thèse : « Né en 1856 à Paris, mais d'origine évidemment alsacienne, si ce n'est allemande, il y a en lui de l'allemand avec sa figure rose et blanche, ses yeux à fleur de tête dissimulés par son binocle, ses cheveux ras comme on les porte volontiers quand on les a rares, même en Allemagne, son jugement peu sûr, soit qu'il se prononce sur le fond ou sur la forme. » (AN : $\mathrm{F}^{17}$ 23811).

6. Tels J. Brunhes, C. Vallaux, E. de Martonne, A. Demangeon, R. Blanchard, A. Vacher, R. de Felice...

7. Lettre au recteur ( $\mathrm{AD}: \mathrm{T} 1277)$.

8. Un trou de dix ans sépare cette génération d'une nouvelle brassée de noms: V. Bérard, p. Camena d'Almeida, L. Gallouédec, H. Lorin, L. Raveneau, E. F. Gautier et L. Gobin, H. Schirmer, A. Bernard... - ces trois derniers n'étant pas des Normaliens.

9. Cf. la note finale adressée au directeur de l'École par Fustel de Coulanges au troisième trimestre 1876-1877, où il le classe, «parmi ceux qui désirent faire de l'histoire », en première ligne à côté de Dubois ( «a fait preuve non seulement de beaucoup d'ardeur au travail, mais d'un véritable sens historique, j'augure bien de lui»), Groussard (" est surtout un esprit sage et prudent; il sera un bon professeur »), et Lacour («n'est pas encore assez maître de lui ni assez maître de ses textes »). Il place en deuxième ligne deux autres élèves, Marcou et Dupuy (AN : 61AJ184). Remarquons à titre anecdotique que Auerbach a fait sa première prestation devant Fustel de Coulanges sur un sujet de géographie.

10. $\mathrm{AN}: \mathrm{F}^{17} 23811$

11. Source : Revue de l'Est et $\mathrm{AN}: \mathrm{F}^{17} 23811$. 
12. "Mauvais jugement, mauvais goût, mais grande érudition ", tel est le sens du rapport signé par A. Himly (AN : $\mathrm{F}^{17}$ 23811).

13. Face à L. Drapeyron, le défenseur malheureux de l'École de géographie et d'une agrégation de géographie, se trouvaient P. Dupuy, L. Gallois, le jeune p. Camena d'Almeida, et Vidal de la Blache, président de séance.

14. $A N$ : $\mathrm{F}^{17} 23811$.

15. AD : W 952/1.

16. Ibid., 17 décembre 1886

17. Un premier libellé, corrigé sur le registre des délibérations, rappelait « la place chaque jour plus grande prise par la géographie dans l'enseignement soit des sciences historiques, soit dans...» (la relation avec l'histoire, est donc abandonnée dans le libellé final adressé au Ministère ; on peut penser que, l'historien Diehl étant le secrétaire de séance, la rectification est due à l'intervention d'Auerbach) (AD : W 952/1).

18. AN : $\mathrm{F}^{17} 23811,1907$

19. Lors de sa succession, en 1926, R. Capot-Rey estime qu'il faut enrichir tout cela : «C'est avec un zèle admirable qu'il réorganisa et modernisa cet enseignement, développant les collections cartographiques, enrichissant la bibliothèque, et groupant surtout un nombre croissant de disciples dont le zèle était attesté chaque année par la soutenance de plusieurs diplômes. » (Annales de l'Est, 1936).

20. Cf. les rapports sur les diplômes publiés dans les Annales de l'Est et adressés au recteur.

21. En 1890-1891 Auerbach adopte le vocable de «chorographie » pour désigner son dessein et son œuvre, plutôt que celui de "chorologie» (pédant), mais il leur préfère l'expression de "géographie régionale» en 1893, abandonnant définitivement une "terminologie pseudogrecque si chère aux Allemands ». Nous préférons utiliser ici science «chorologique " par référence à la position épistémologique d'Hettner que Auerbach commente avec sympathie en 1909 , et pour éviter une confusion entre option « régionale » et opinion « régionaliste ».

22. Elle a été subventionnée par les départements de Meurthe-et-Moselle et des Vosges, la municipalité de Nancy, les Amis de l'Université.

23. Le rapport est signé par C. Pfister, président du jury, mais on peut penser qu'il reprend la discussion menée par Auerbach. C'est nous qui soulignons.

24. XIII ${ }^{\mathrm{e}}$ congrès national des sociétés savantes de géographie, Nancy 1901, Bulletin de la Société de géographie de Rochefort, 1902, p. 1-20.

25. Bulletin de l'Université populaire de Nancy (1802-1808), livraison de mars 1902, compte rendu signé Em.N.

26. Le rôle potentiel de capitale régionale n'est pas exploré par les publications liées à la Société industrielle de l'Est que le géographe Louis Laffitte anime en tant que secrétaire dans les années dix. C'est dans La France de l'Est de Vidal de la Blache (1917) que se produit cette promotion de Nancy comme métropole moderne (Ozouf-Marignier, 1998 et recherches en cours), puis, à la Chambre de Commerce, dans les perspectives de la réforme Clémentel de 1917. On connaît mal les relations entre Auerbach et cette Société très fréquentée par les scientifiques (Birck, 1998).

27. AN : 61AJ184.

28. Cf. l'éditorial de la brochure «L'Éducation sociale» (octobre 1906) qui rappelle les idéaux d'un mouvement développé dans le sillage de l'Affaire Dreyfus.

29. La partie Alsace-Lorraine disparaissant bien sûr dans la Bibliographie 1915-1919 publiée en 1921.

30. Compte effectué pour la seule période d'avant-guerre.

31. La rubrique «Allemagne » est partagée entre quelques contributeurs.

32. Plusieurs témoignages suggèrent que les géographes de la génération d'Auerbach étaient jugés dépassés par la jeune génération.

33. $A N: F^{17} 23811$. 


\section{RÉSUMÉS}

Auerbach est l'un des premiers Normaliens historiens qui se sont convertis à la géographie. L'article le situe par rapport aux premières générations qui ont contribué à l'essor universitaire de la discipline. Il montre par quels choix institutionnels et épistémologiques Auerbach a réussi à autonomiser la discipline dans la chaire de géographie qu'il a occupée à la faculté des lettres de Nancy de 1893 à 1926 : comme le groupe des Annales de géographie, il a défendu une conception chorologique de la discipline. L'article souligne aussi les singularités de sa personnalité et sa forte implication dans le régionalisme.

Auerbach is one of the first graduates of the Ecole Normale Supérieure (Paris) who converted to Geography. The article places him in relation to the first generations which contributed to the rapis university expansion of the discipline. It shows by which institutional and epistemological choices, Auerbach succeeded in autonomising the chair of Geography which he occupied at the Faculty of Letters at Nancy from 1893 to 1926 : like the Annales de géographie group, he defended a regional conception of the discipline. The article underlines also the singularities of his personality and his strong susceptibility to regionalism.

Auerbach ist einer der ersten Absolventen in der Geschichte der Ecole Normale Supérieure (Paris), die sich zur Geographie bekannten. Als Lehrstuhlinhaber in Nancy (1893-1926) verteidigte er eine regionale Auffassung der Geographie. Er zeigte sich sehr empfänglich für einen Regionalismus.

\section{INDEX}

Schlüsselwörter : regionale Geographie, Regionalismus, Universität Nancy

Keywords : generation, regional geography, regionalism, université populaire, university of Nancy

Mots-clés : génération, géographie régionale, régionalisme, université de Nancy, université populaire

\section{AUTEUR}

\section{MARIE-CLAIRE ROBIC}

Directeur de recherches (CNRS), Épistémologie et histoire de la géographie - 9 rue Malher 75181 Paris Cedex 4 